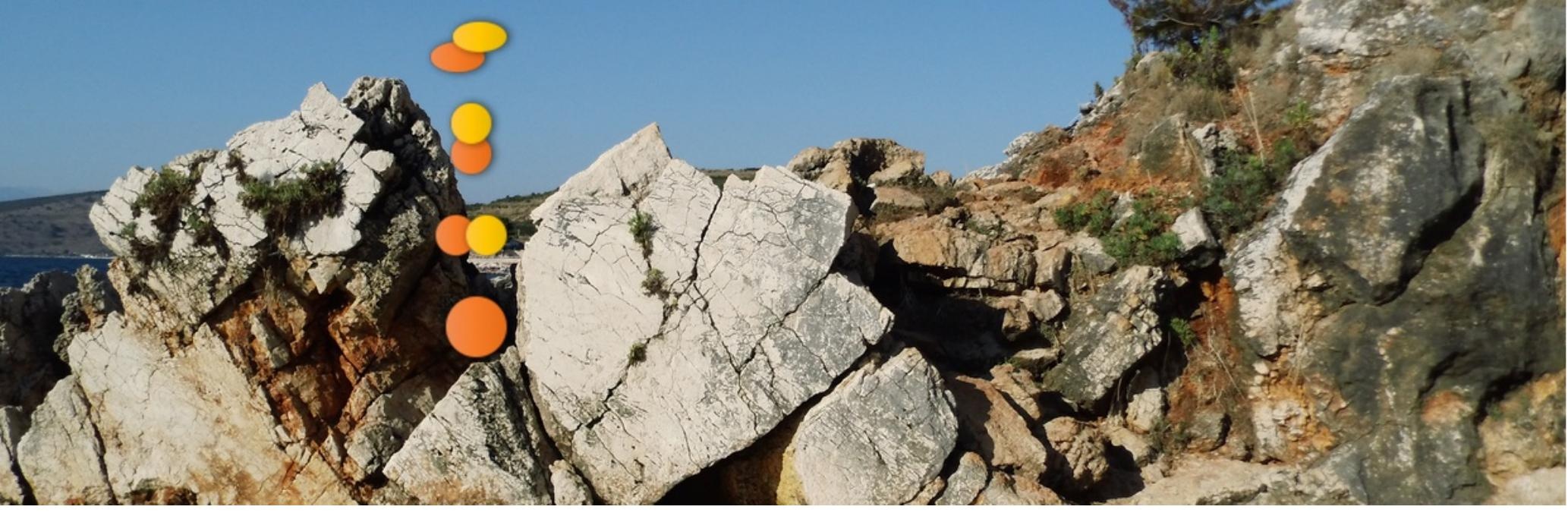

Hybrid Structures as a Symbiotic Bond of Art and Science

$\begin{aligned} \text { Authors: } & \text { Bujar Q. Bajçinovci } \\ \text { Submitted: } & \text { 23. September } 2016 \\ \text { Published: } & 1 . \text { October } 2016 \\ \text { Volume: } & 3 \\ \text { Issue: } & 5 \\ \text { Keywords: } & \text { Hybrid Structures, Architecture, Art, Science, Symbiosis } \\ \text { DOI: } & 10.17160 / \text { josha.3.5.233 }\end{aligned}$

Journal of Science, Humanities and Arts

JOSHA is a service that helps scholars, researchers, and students discover, use, and build upon a wide range of content 


\title{
Hybrid Structures as a Symbiotic Bond of Art and Science
}

\author{
Bujar Bajçinovci \\ University of Prishtina, Faculty of Civil Engineering and Architecture, Kosovo. \\ Email: bujar.bajcinovci@uni-pr.edu
}

\begin{abstract}
The world continues to expand its development in all possible spheres, and this expanding is in an arithmetic mode of progression, hence, as well the population continues to grow. So, in terms of urban dynamics, sustainable architecture and artistic challenges as presents this phenomenon, we need more environmental and sustainable clear objectives. What are hybrid buildings? What do they present? Currently, the potential for hybrid buildings has taken a real evolutive urban concept, in relation, and as a consequence of economics, lack of open spaces, spatial contemporary concepts with the urban prerequisites has increased the overall value of urban future zones. This paper explores the design concepts of structured hybrid buildings as a creative interdependence between science, art, architecture and human development. Hence, in addition this paper explores the possibility of urban patterns and trans functional compositions of structured hybrid buildings. Structure and functionality of future hybrid buildings will be manifested with many possibilities, moreover we argue that within a few decades a more fundamental step in human development will occur, especially in architecture, art and science. Moreover, there is a great potential for further development of future contemporary trends, on architectural structures, with different conceptual objectives, mentalities, habits and the style of living? Art, architecture, science, and human development, hereafter will need a more symbiotic bond! This merge, this new symbiosis of hybridization, will generate a new identity of architectural distinctiveness, and different features will reappear at certain levels of functionalities by which are linked to contentious interdependence of each other. Thus, we conclude that the hybrid building structures present unique challenges with overall varieties, which are both possible and desirable, therefore, hybrid building structures can evolve in different dimensions as per today presented. Ergo, we argue that hybrid building structures will offer more sustainable affinities between: profit, cultural, social, and human development in coming decades.
\end{abstract}

Keywords: Hybrid Structures, Architecture, Art, Science, Symbiosis

\section{INTRODUCTION}

The world continues to expand its development in all possible spheres, and this expanding is in an arithmetic mode of progression, hence, as well the population continues to grow. So, in terms of urban dynamics, sustainable architecture and artistic challenges as presents this phenomenon, we need more environmental and sustainable clear objectives. Thus, on this matter and in regard for sustainable development we need to find acceptable answers, to the primary meaning of those fundamental challenges. In terms of urban planning and urban design, the first and the most valuable objective is demand for quality housing and public health. Moreover, in addition, we also have urgency for: commercial business areas, 
green dedicated and cultivated areas, industry zones with less pollution. All these requirements are direct reflected in our health, and style of living. The most crucially, those contemporary developments and features are querying to be answered in an adequate and sustainable response. The actual phenomenon's and the new style of living, especially, have great impact on overall human culture and communities, demanding to be applied new strategies and action plans which will be primary oriented on quality of life. Ergo, those actions must be immediate and irreplaceable policy for a healthier environment or umwelt. Therefore, as a consequence there is a need to be gradually redefined the design concepts, as applied and established per to date. In this regard, we argue that adequate attention should be given to the hybrid multiuse buildings. What are hybrid buildings? What do they present? Currently, the potential for hybrid buildings has taken a real evolutive urban concept, in relation, and as a consequence of economics, lack of open spaces, spatial contemporary concepts with the urban prerequisites has increased the overall value of urban future zones. Hence, the phenomenon of globalisation has growth recognition of so far wealth lacked regions, in addition, the contemporary styling of living, the current cultural trends and the concept of values, additionally has been changed! This growth of the new trends among artists has established a new cultural, humanitarian, and personal values. There are no more, avant-garde taboos, ergo, more of those cultural and artistic challenges meet the conceptual findings of the contemporary science, science and art are furthermore bonding. All architectural designs actually are challenged with fundamental questions: how can we live more cultural, social, sustainable and healthier life in coming decades or centuries? As far as architecture is concerned, there is a physiological gap, a commitment and necessity, that architects have to re-find themselves, associated with resurgence of transdisciplinary new professions. A multiuse, and multidimensional structures with a potency of notions such as holistic breathing, operated as a more of 'the sum of all parts', or mixed functions. Prospective structures which will reflect transdisciplinary professions.

Fenton stated that: "there is a difference between mixed-use and hybrid buildings. He argued, the individual programs of hybrid buildings relate to one another and begin to share intensities. Hybrid buildings can be compared to a genetic cross breed of different species" $[1,2]$. Moreover, Fenton argued that hybrids structures just from the beginning of the $20^{\text {th }}$ century was a conceptual idea in urban planning as an attempt to modernize United States Cities, ergo, a more contemporary actions reflected on urban design to reformulate the new urban zones. There was a great meditation on urban planning regarding to the multi-use of 
new buildings. Also, many designers were also involved in holistic understanding to optimize and reevaluate the planed urban regions, moreover, building designs was anticipated to transform relationships between urbanites and a future perception of the new way of living. A more sustainable and healthier life style approaches, regarding to the more complexed urban and social forthcomings.

"Generally, in regard to functional brief, hybrid buildings are characterized by a high programmatic complexity. These can incorporate different categories of urban uses, allowing a great capacity of adaptation and interaction with the urban environment they integrate, containing unique and distinct characteristics from other buildings of this type in different urban contexts" $[3,1]$.

Similarly, the conceptual artistic and architectural ideas related to the hybridization presents more than just one program, those ideas involve a transdisciplinary symbiosis of disciplines with a clear objective: "responding to three major concerns of our society" [2]:

- "The land scarcity and its high value, the need of intensifying land use in order to contribute to sustainable development and the need of densifying uses in order to revitalize urban centers" [2].

- "The need for specificity is allowing some architects to engage program rather than simply building for maximum (and Generic) flexibility, as was the predominant case previously" [2].

- "Land and construction cost with conservative approaches by government on spending for public infrastructure has forced many civic institutions to find new ways of housing and funding themselves" [2].

From all the states above, we can argue that the multiuse buildings or hybrid multifunctional buildings offer a fundamental unfolding relationships to the contemporary market requirements and profit. A profit, as a generator of social, and cultural development, moreover, actually we are witnessing the demands of those design concepts in Kosovo, a market pressure, wishes for those types of buildings are more than obviously. Hence, we are in a complex situation, actually, development of social phase of Kosovar communities are urging the necessity of growing fiscal state budget, gross domestic product, supply and demand in market determination, quality housing, and public heath demands are in contentious interdependence. 
Hence: Does concepts of hybrid buildings can offer a more sustainable affinity between cultural, social, sustainable and healthier life in coming decades?

\section{MATERIALS and METHODS}

This paper explores the design concepts of structured hybrid buildings as a creative interdependence between science, art, architecture and human development. Moreover, this paper investigates the possibility of urban patterns, and trans-functional compositions of new structured hybrid buildings. The research methods consist of empirical observations to the need of communities and urbanites of Kosovo, with methods of theory analysis, hence, with an emphasised focus to the present urban structure of the capital of Kosovo, Prishtina. In form to receive a clear and exceeding information's, research is made within urban frameworks, shapes of architectural and urban structures, and the possibilities of expanding directions of the city of Prishtina, regarding to the spatial morphologies of shapes which compose the city silhouette. Hybrid buildings were investigated through literature review, documentations, drawings, and sketches. More books and research papers are consulted, articles, maps and old pictures. The supplementary data for this study is explored and sorted by the possibilities of spatial urban planning of city Prishtina regarding to the future concepts of Design Build needs, and promoted-accepted future design strategies of Municipality of Prishtina.

Cities are complex ecosystems with specific phenomenon's directly reflected in our health, natural resources, economics, social, and aesthetic fields. They are open integrated systems, and huge organisms with specific and complex metabolism that transform vast amount of energy, generate huge amount of waste, and emanate a number of specific environmental not so appropriate impacts. However, it can be conceptually considered, that cities are locally and regionally specific, and therefore the negative environmental emanations are authentic activities, and specific attributes of each city [4]. Environmental design strategies must incorporate holistic, and bionomics objectives:

- We must always explore solutions and symbiotic harmonies between the environment, location, architecture and buildings.

- We should encourage buildings which could operate, and offer services 24/7.

- We must always explore solutions and symbiotic harmony between the 'horizontality and verticality' of erected structures, and indwelling's. 
- We should encourage urban structures with complex functions and purposes, preferred with diversity, interconnected and dynamic functional relationships.

Hence, The General Assembly of UN, in: "Recalling its resolution 64/236 of 24 December 2009, in which it decided to organize the United Nations Conference on Sustainable Development at the highest possible level in 2012, as well as its resolution 66/197 of 22 December 2011", "Calls on the future We Want" [5]:

- "We therefore acknowledge the need to further mainstream sustainable development at all levels, integrating economic, social and environmental aspects and recognizing their interlinkages, so as to achieve sustainable development in all its dimensions" [5].

- "We recognize that poverty eradication, changing unsustainable and promoting sustainable patterns of consumption and production and protecting and managing the natural resource base of economic and social development are the overarching objectives of and essential requirements for sustainable development. We also reaffirm the need to achieve sustainable development by promoting sustained, inclusive and equitable economic growth, creating greater opportunities for all" [5].

- "We recognize that people are at the centre of sustainable development and in this regard we strive for a world that is just, equitable and inclusive, and we commit to work together to promote sustained and inclusive economic growth, social development and environmental protection and thereby to benefit all" [5].

Urban planning and the vision of future growth of the city's is characterized by the demand for services which efficiently increases the capacity of functionalities, meaning, as a whole ecosystem, with its basic urban services, and municipals management, as: Energy, Water and Sanitation, Heating, Public Health and Environmental friendly sub-urban areas. Fast growing cities have specific emergency needs, characterized with new specific urban challenges, often, with completely different development demands. Conceptually, there is not an urgency to declare that we should always consider to design a 'new buildings' with multiuse functions immediately. Hence, there is always a possibility to encourage the urban strategy for revitalization of buildings and urban blocks instead, when this possibility encourages, and promote environmental sustainability. This conceptual holistic strategy 
additionally supports the contemporary objectives of science, art, and human development. Ergo, those hybrid urban structures preferably must find itself on urban patterns, like:

- These buildings often are presented as interpolation of mixed functional parts.

- As functional demand for urban blocks, regarding to the spatial development.

- Those structures often form a cross functional, and space bridges.

- As a part of revitalisation of old and ruined buildings.

- As a new expanding structure for future businesses.
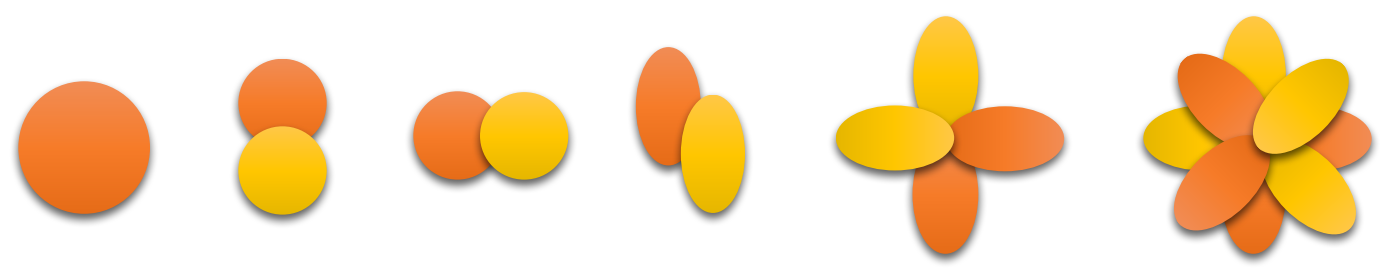

Figure 1. Shapes which functionally evolve, and transforms accordingly to hybrid multifunctional zones. (Source: Author, 2016).

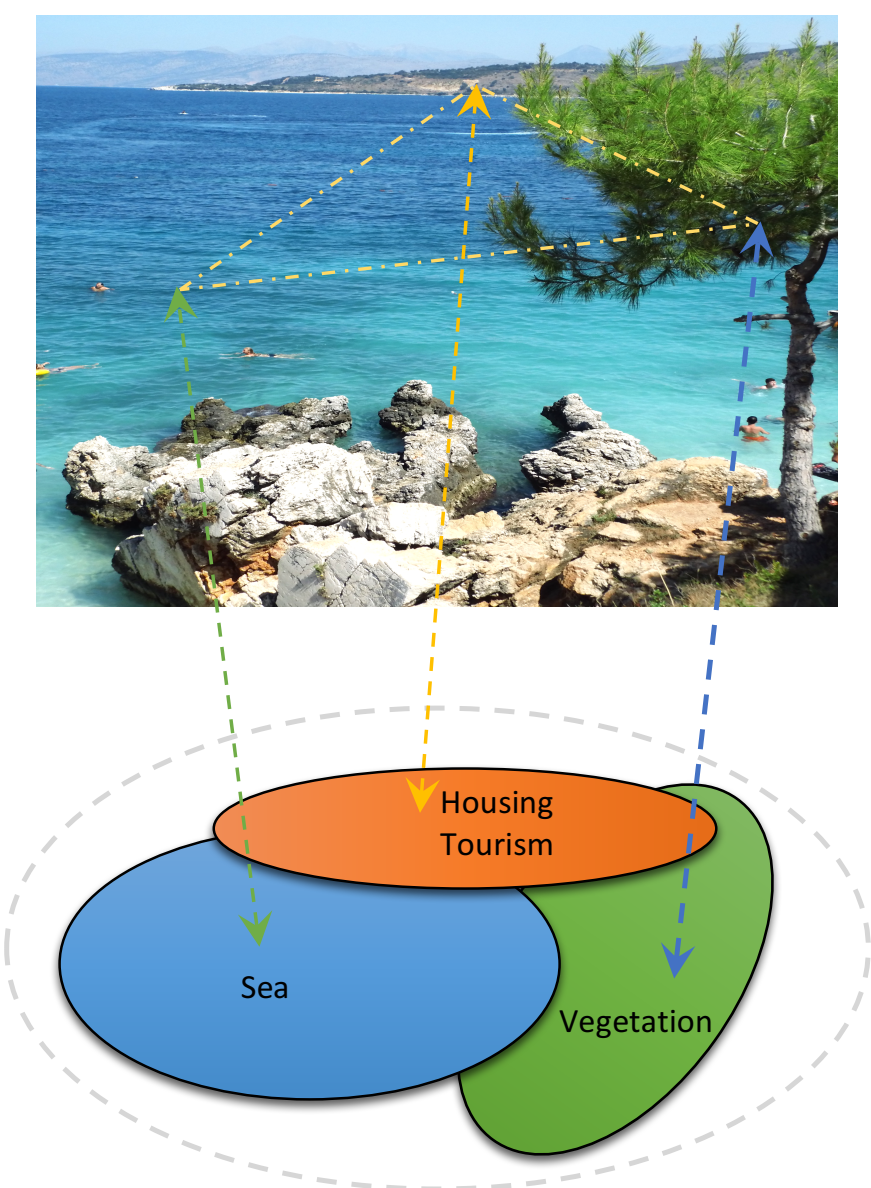

Figure 2. Functional symbiosis of nature and societies: Water, Housing with Tourism, and Vegetation. (Source: Author, 2016). 


\section{RESULTS and DISCUSSION}

Structure fluid dynamics, and organic multiuse buildings are the possible future objectives of environmental sustainable design. Furthermore, structure and functionality of future hybrid buildings aim many possibilities, in this context, we argue that within a few decades a more fundamental step in human development will occur, especially in human development, art, and science. Moreover, there is a great potential for further development of future contemporary trends on architectural structures, with different conceptual objectives, mentalities, habits and the style of living? Art, human development, science, urban culture, and society concepts will need a more symbiotic bond! This merge, this new symbiosis of hybridization, will generate a new identity of architectural distinctiveness, and different features will reappear at certain levels of functionalities by which are linked to contentious interdependence of each other. A bonding process of art and science reflected on hybridisation of structures, forms, functionalities, and uniqueness's of architecture interpretation.

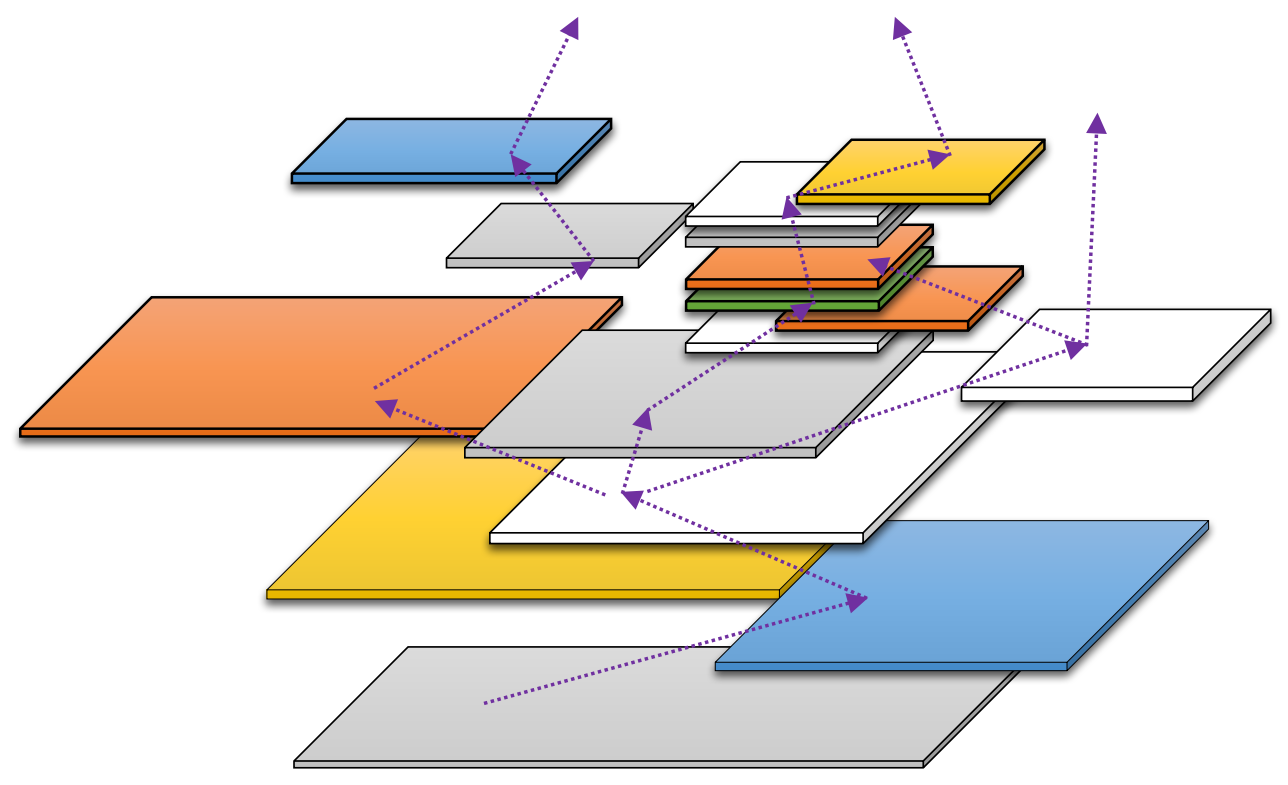

Figure 3. Decomposition of horizontal and vertical zones, art and architectural space wholeness's. (Source: Author, 2016).

Ergo, we must constantly explore for real life functional and structural solutions, in order to manifest co-created values of hybridisation, emphasising to the values of economics and rentability, furthermore, we must carry attention to the commonly named 'full time job' building structures. The 'amalgamation' of more than two functions, can potentially result in a more environmental adaptability, and self-sustain profitability of new architectural structures. 

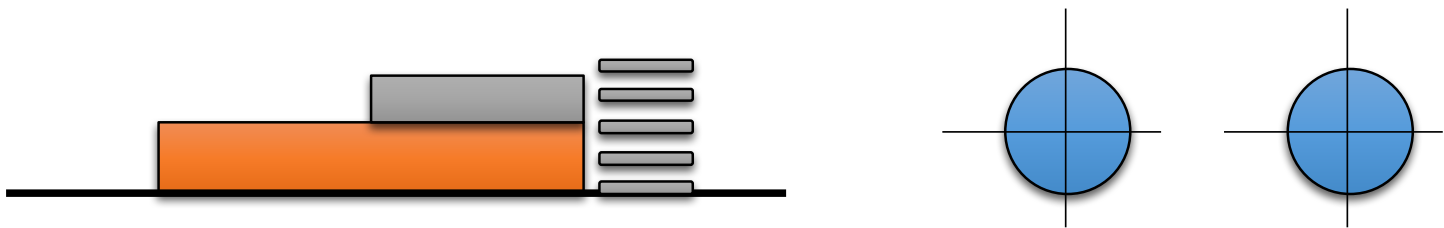

Figure 4. Architectural structures with a 'full time job', 24/7, and rentable economics. (Source: Author, 2016).
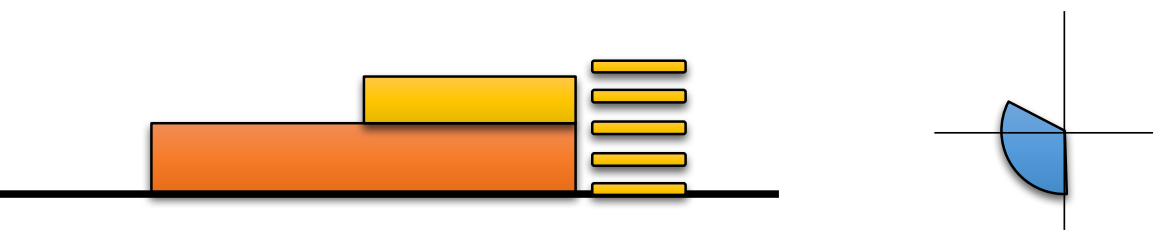

Figure 5. Architectural structures (like Operas), with a $4^{\mathrm{h}}$ service in day, week, or a month. (Source: Author, 2016).

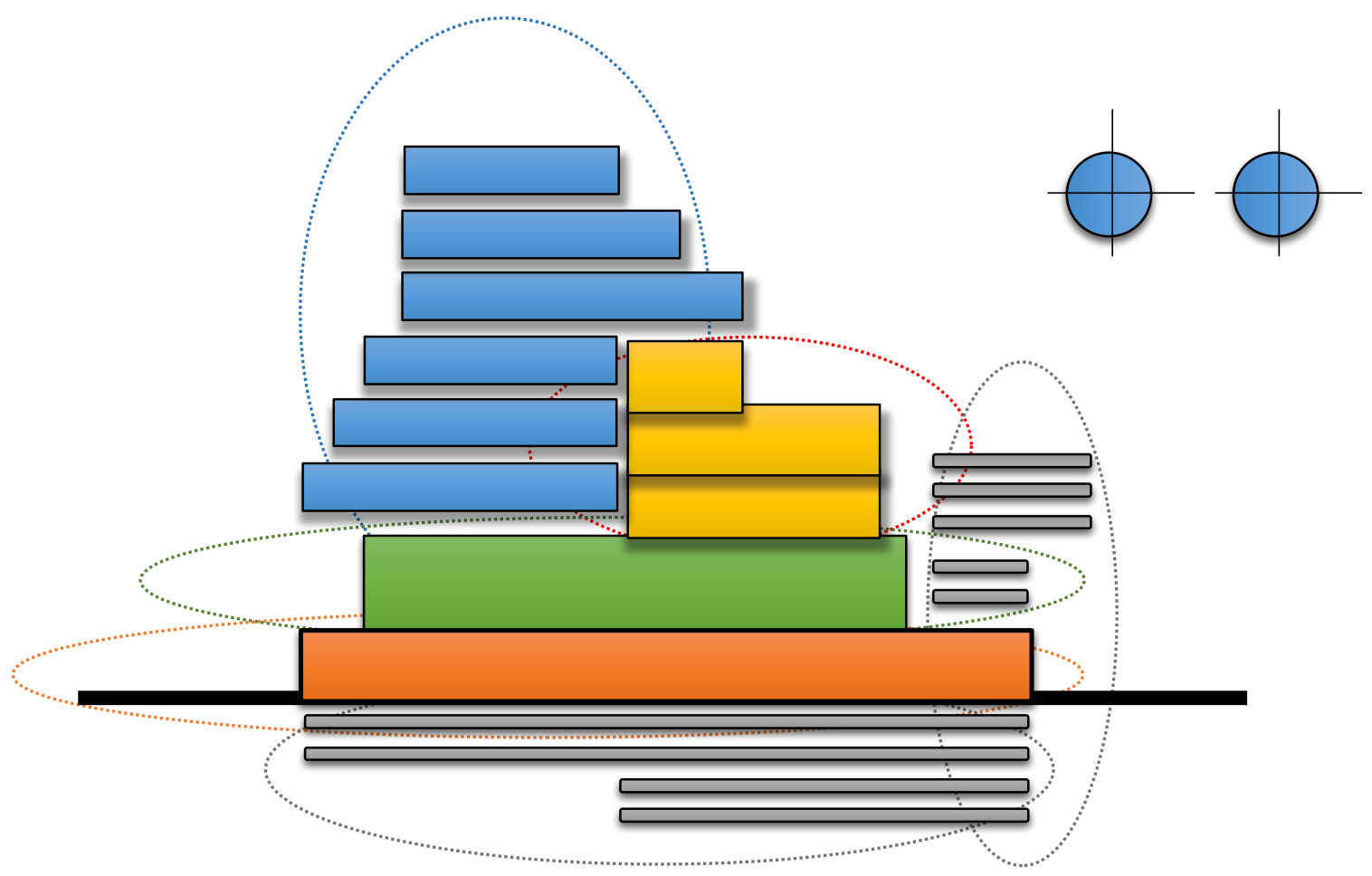

Figure 6. Multi-functional pattern, a more iterations, finding a more diversities in hybrid structures. (Source: Author, 2016). 

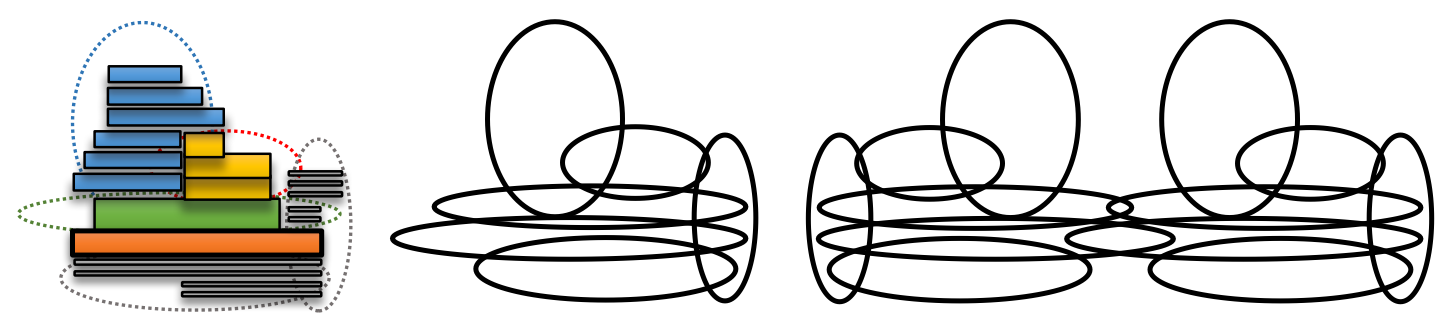

Figure 7. Patterns with a more iterations, finding possibilities: profit, shapes, and functional diversities in hybrid structures. (Source: Author, 2016).
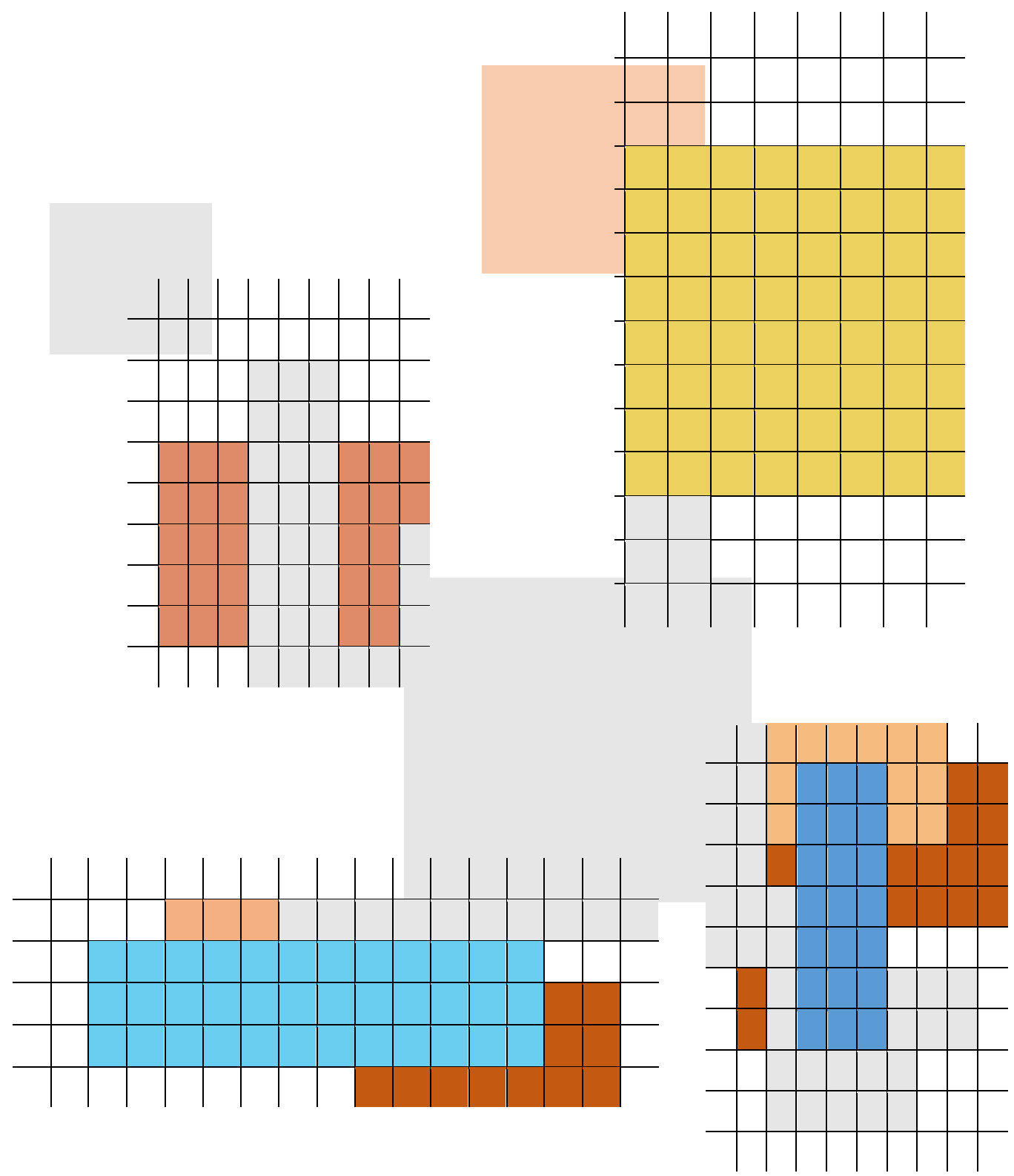

Figure 8. Functional modular zones, seeking attributes of: proportions, relations, dependencies, art expression, form, volume in relation to a successful hybridization. (Source: Author, 2016). 

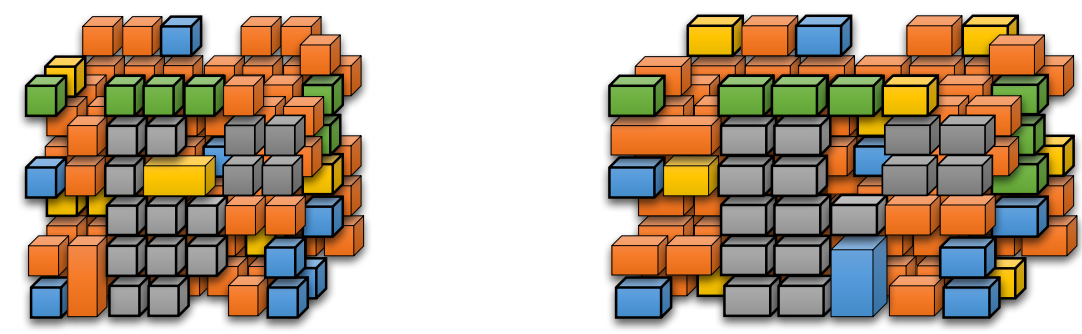

Figure 9. 3D modular iterations, varieties, seeking hybridization. (Source: Author, 2016).

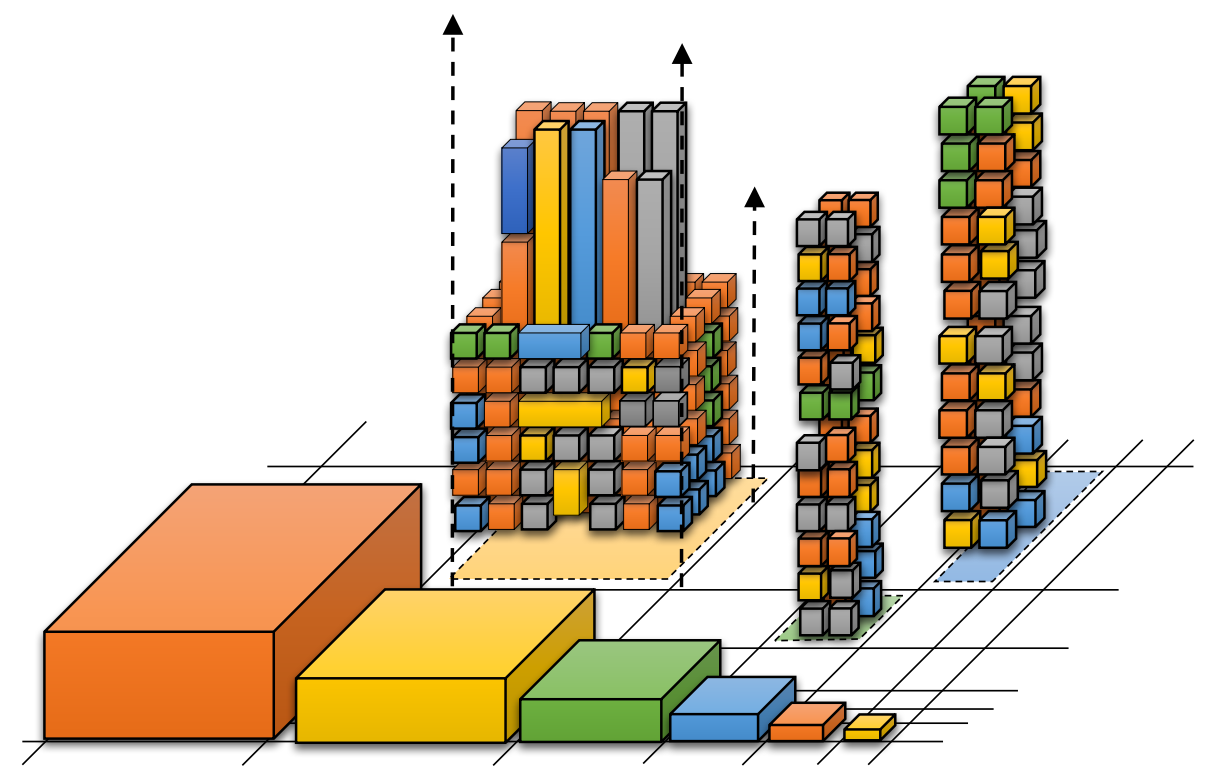

Figure 10. Chosen modular zones, for conceptual hybridization. (Source: Author, 2016).

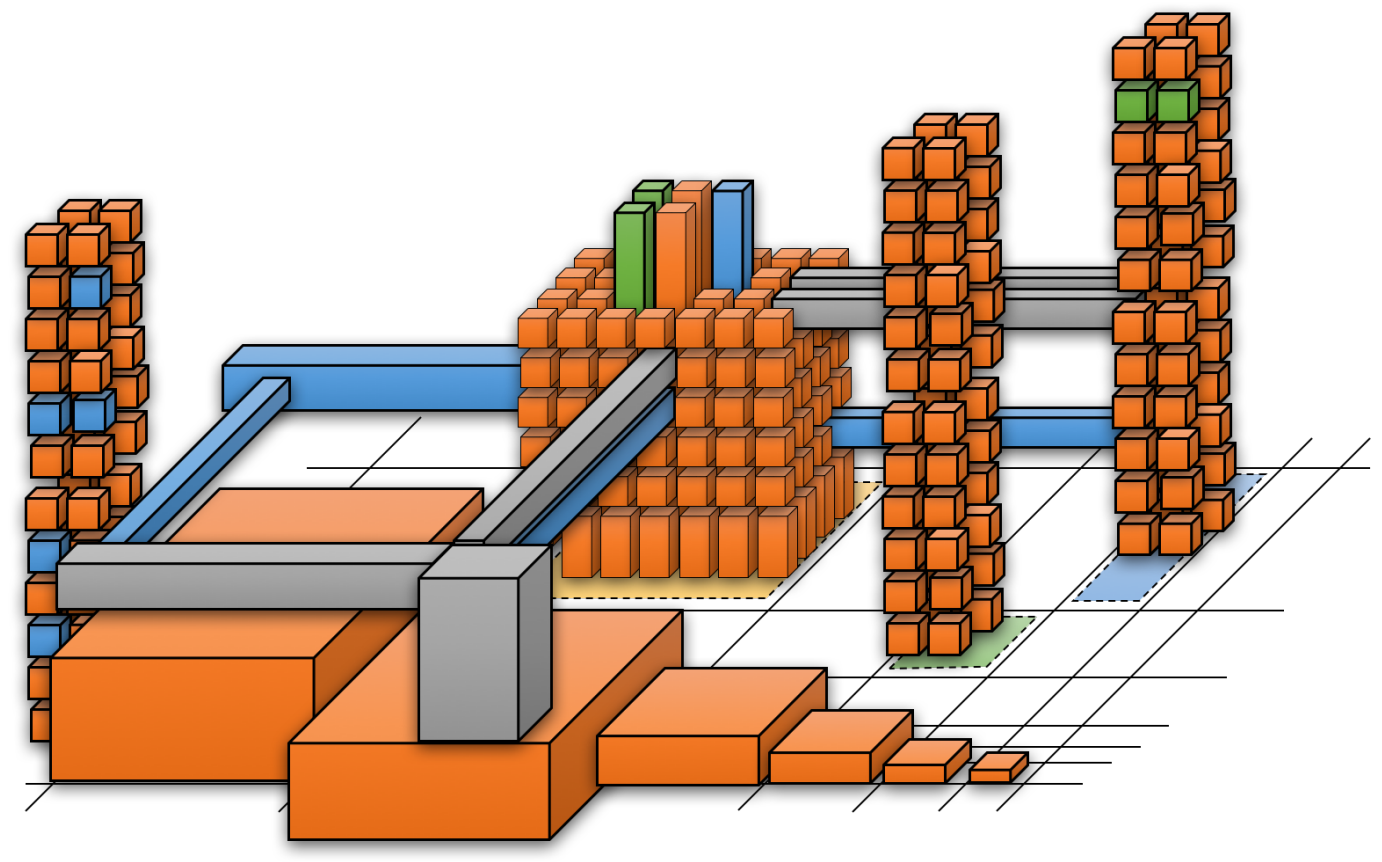

Figure 11. Preferred final concepts of modular hybridization. (Source: Author, 2016). 


\section{CONCLUSIONS}

Structure fluid dynamics, and organic multiuse buildings are the possible future goals of environmental sustainable design. Furthermore, structures and functionalities of future hybrid buildings will be manifested with many possibilities, moreover we argue that within a few decades a more fundamental step in human development will occur, especially in architecture, art and science. Moreover, there is a great potential for further development of future contemporary trends of architectural structures, with different conceptual objectives, mentalities, habits and the style of living? Ergo, we argue that art, human development, science, urban culture and society concepts, hereafter will need a more symbiotic bond! This merge, this new symbiosis will generate a new identity of artistic, and urban attributes, hence, different features will reappear at certain levels of functionalities by which are linked to contentious interdependence. A bonding process which will more merge art and science, a impression on hybridisation of structures, profit, form, functionalities, and uniqueness's of architecture interpretation. The preferred methods for hybrid structures, stated above are quite evolutive, as to be used for further development and research. With the society moving towards greater energy demand in proportion with degraded environmental sustainability, we must reconsider to adapt and change. Hence, we conclude that the hybrid building structures present unique architectural challenges with many varieties, which are both possible and desirable. Moreover, hybrid building structures can evolve in different dimensions as per today presented, merging more affinities between art, science, and human development in coming decades.

\section{References}

[1] Fenton, J. (1985). Hybrid Buildings. Princeton Architectural Press.

[2] Byrd, B. (2014). Hybrid Buildings. ENVD 3300: $\quad$ Green Neighbourhoods Praxis. Instrutors: Michael Tavel + David Kahn. Spring 2014. http://www.colorado.edu/envd/sites/default/files/attachedfiles/Hybrid\%20Buildings Byrd.pdf [Accessed: September, 2016].

[3] Sofia, Felisberto das Neves, A. (2012). Residential Hybrid Buildings Different Temporalities in City's life. Instituto Superior Tecnico, Universidade Tecnica de Lisboa. https://fenix.tecnico.ulisboa.pt/downloadFile/395144950900/resumo\%20alargado \%20FINAL.pdf pp, 1-2.

[Accessed: September, 2016].

[4] Bajçinovci, B. (2012). PhD Thessis: Integrated Design Process of Public and Residential Buildings: Transdisciplinary Approach on Evolutionary Design. University of Sarajevo, BiH.

[5] UN, (2011). Future We Want-Outcome document. Sustainable Development. United Nations Department of Economic and Social Affairs. https://sustainabledevelopment.un.org/futurewewant.html [Accessed: September, 2016]. 